\title{
SAT-solving Based on Boundary Point Elimination
}

\author{
Eugene Goldberg, Panagiotis Manolios \\ Northeastern University, USA \{eigold, pete\}@ccs.neu.edu
}

\begin{abstract}
We study the problem of building structure-aware SAT-solvers based on resolution. In this study, we use the idea of treating a resolution proof as a process of Boundary Point Elimination (BPE). We identify two problems of using SAT-algorithms with Conflict Driven Clause Learning (CDCL) for structure-aware SAT-solving. We introduce a template of resolution based SAT-solvers called BPE-SAT that is based on a few generic implications of the BPE concept. BPE-SAT can be viewed as a generalization of CDCL SAT-solvers and is meant for building new structure-aware SAT-algorithms. We give experimental results substantiating the ideas of the BPE approach. In particular, to show the importance of structural information we compare an implementation of BPE-SAT and state-of-the-art SAT-solvers on narrow CNF formulas.
\end{abstract}

\section{Introduction}

In the last decade, SAT-algorithms have seen a great deal of success in solving real-life formulas. (Since the key idea of modern SAT-algorithms is Conflict Driven Clause Learning we will refer to them as CDCL SAT-solvers.) This success can be attributed (among other things) to using effective heuristics controlling various aspects of the behavior of a CDCL SAT-solver such as decision making, removal of obsolete conflict clauses and so on. The problem, however, is that modern SAT-solvers are based on the resolution proof system that is most likely non-automatizable [1]. Informally, this means that there is no efficient deterministic procedure for finding short resolution proofs. So even if a class of formulas has short proofs, a deterministic SAT algorithm may not be able to find them and hence may have very poor performance. For example, CDCL SAT-solvers often display abysmal performance on formulas describing equivalence checking of two identical copies of combinational circuits despite the existence of linear size resolution proofs for such formulas.

A natural way to cope with non-automatizability of resolution is to provide a SAT-solver with additional information about the formula structure. In this paper, we study the problem of building structure-aware resolution-based SATsolvers. We use the approach of [8] where a resolution proof is considered as a process of Boundary Point Elimination (BPE for short). Our intent here is to point out the problems with building structure-aware CDCL SAT-solvers and suggest a direction for future research. 
Given a CNF formula $F$, an $l$-boundary point is an unsatisfying complete assignment $\boldsymbol{p}$ such that all clauses of $F$ falsified by $\boldsymbol{p}$ have literal $l$. The elimination of $\boldsymbol{p}$ as a boundary point is to add a resolvent $C$ of clauses of $F$ such that $\boldsymbol{p}$ is not a boundary point of $F \wedge C$. Importantly, in any resolution proof, all boundary points of $F$ have to be eliminated. An $l$-boundary point (where $l$ is a literal of variable $x_{i}$ ) is eliminated only by particular resolutions on $x_{i}$. So boundary points of $F$ specify "mandatory" resolutions.

We describe a generic SAT-solver called BPE-SAT that is based on the following four observations. First, any resolution-based SAT-algorithm has to generate mandatory resolutions. Second, it is preferable to find such resolutions early since they may make many non-mandatory resolutions redundant, which allows one to find a proof faster and make it smaller. (In particular, an entire proof may consist only of mandatory resolutions e.g. for equivalence checking formulas.) Third, finding an $l$-boundary point of $F$ is hard (it reduces to a SAT-check for the formula $F \backslash\{$ the clauses of $F$ with literal $l\}$ ). Fourth, if $\boldsymbol{p}$ is an $l$-boundary point of $F, F^{\prime}$ is a subset of clauses of $F$ and $F^{\prime}(\boldsymbol{p})=0$, then $\boldsymbol{p}$ is an $l$-boundary point of $F^{\prime}$. So by eliminating $l$-boundary points of $F^{\prime}$ one also eliminates $l$ boundary points of $F$ (and if $F^{\prime}$ is small, finding boundary points of $F^{\prime}$ is much easier than for $F$ ).

BPE-SAT repetitively extracts a subformula $F^{\prime}\left(X_{1}, X_{2}\right)$ of $F(X)$ (where $X_{1}, X_{2}$ are non-overlapping subsets of $X$ ) and then existentially quantifies away the variables of $X_{1}$ producing clauses depending on variables of $X_{2}$. Existential quantification of $F^{\prime}$ is performed by an algorithm called the BPE procedure. To quantify away a variable $x_{i} \in X_{1}$, the BPE procedure adds to $F^{\prime}$ resolvents that eliminate all symmetric $x_{i}$-boundary and $\bar{x}_{i}$-boundary points. The BPE procedure eliminates variables of $X_{1}$ one by one and so works similarly to the DP algorithm [6]. However, we believe that relaxing the order in which boundary points are eliminated may lead to developing a much more powerful procedure of existential quantification.

BPE-SAT is based on a few very general observations listed above and so, is formulated as a template of a resolution based SAT-solver. In particular, we do not specify in BPE-SAT how a subformula $F^{\prime}$ is extracted from $F$. This is done for the following reason. According to the observations above, the choice of subformula $F^{\prime}$ is good if the latter shares boundary points with $F$. Intuitively, the complexity of identifying good subformulas $F^{\prime}$ is what makes resolution non-automatizable (the choice of $F^{\prime}$ strongly depends on the structure of $F$ ). So, in this paper, we rather formulate the problem of finding subformulas $F^{\prime}$ that share boundary points with $F$ than solve it. Interestingly, our definition of good subformulas gives a new meaning to the notion of the formula structure in the context of resolution proofs. (The structure of formula $F$ is specified by a set of its subformulas sharing boundary points with $F$ ).

A natural question is how BPE-SAT is related to CDCL SAT-solvers. Remarkably, despite BPE-SAT being just a theoretical construction, a CDCL SATsolver is one of (many) implementations of BPE-SAT. The set of clauses responsible for a conflict specifies the subformula $F^{\prime}$ to be selected from $F$. Existential 
quantification of variables of $F^{\prime}$ is performed by the conflict clause generation procedure. The latter can be represented as the BPE procedure eliminating boundary points specified by the partial assignment leading to the conflict.

Another important issue is whether BPE-SAT brings in something new with respect to CDCL SAT-solvers. Both BPE-SAT and CDCL SAT-solvers are based on resolution and it was recently shown that resolution proofs can be polynomially simulated by non-deterministic CDCL SAT-solvers [9]. It is tempting to use these results for optimistic predictions about the performance of deterministic CDCL SAT-solvers. However, there are at least two problems that dampen this optimism. (The introduction of BPE-SAT is a way to address these problems.) The first problem is that eager backtracking of a CDCL SAT-solver (i.e. after a conflict occurs) may lead to generation of subformula $F^{\prime}$ (specified by the conflict) that does not share boundary points with $F$. This issue is addressed in BPE-SAT by posing the problem of finding subformulas $F^{\prime}$ sharing boundary points with $F$. The second problem is that a SAT-solver based only on conflict driven learning cannot efficiently quantify away the variables of $X_{1}$ from subformula $F^{\prime}\left(X_{1}, X_{2}\right)$ (even if the resulting set of clauses depending on variables of $X_{2}$ is small). This problem is totally ignored by the results of [9] because it is caused by the difference in termination conditions of non-deterministic and deterministic CDCL SAT-solvers when performing existential quantification. (And [9] is concerned only with non-deterministic CDCL SAT-solvers). In BPE-SAT, this problem is addressed by showing the promise of performing existential quantification using boundary point elimination.

The contributions of this paper are as follows. First, we introduce a template SAT-solver BPE-SAT and justify this introduction in terms of BPE. Second, we identify some problems of CDCL SAT-solvers in building structure-aware SAT-solvers and point out directions for solving those problems. In particular, we show that BPE can be used for existential quantification. Third, we give an example of tuning BPE-SAT to a particular class of formulas (narrow formulas).

\section{Basic Definitions}

In this section, we recall some basic definitions of SAT solving.

Definition 1. A literal $l\left(x_{i}\right)$ of a Boolean variable $x_{i}$ is either $x_{i}$ itself or its negation (denoted as $\bar{x}_{i}$ ). We will also denote a literal as just $l$ in the contexts where the identification of $x_{i}$ can be omitted. A clause is the disjunction of literals where no two (or more) literals of the same variable can appear. $\boldsymbol{A} \boldsymbol{C N F}$ formula is the conjunction of clauses. We will also view a CNF formula as a set of clauses. Denote by $\operatorname{Vars}(\boldsymbol{F})$ (respectively $\operatorname{Vars}(\boldsymbol{C})$ ) the set of variables of CNF formula $F$ (respectively clause $C$ ).

Definition 2. Given a CNF formula $F\left(x_{1}, \ldots, x_{n}\right)$, a complete assignment $\boldsymbol{p}$ (also called $\boldsymbol{a}$ point) is a mapping $\left\{x_{1}, \ldots, x_{n}\right\} \rightarrow\{0,1\}$. A clause $C$ is satisfied (respectively falsified) by $\boldsymbol{p}$ if $C(\boldsymbol{p})=1$ (respectively $C(\boldsymbol{p})=0$ ). 
Definition 3. Given a CNF formula F, a satisfying assignment $\boldsymbol{p}$ is a complete assignment satisfying every clause of $F$. The satisfiability problem (SAT) is to find a satisfying assignment for $F$ or to prove that such an assignment does not exist.

Definition 4. Let clauses $C^{\prime}, C^{\prime \prime}$ have the opposite literals of variable $x_{i}$ (and only of $x_{i}$ ). The resolvent $C$ of $C^{\prime}$ and $C^{\prime \prime}$ on variable $x_{i}$ is the clause with all the literals of $C^{\prime}$ and $C^{\prime \prime}$ but those of $x_{i}$. The clause $C$ is said to be obtained by a resolution operation on $x_{i} . C^{\prime}$ and $C^{\prime \prime}$ are called the parent clauses of $C$.

Definition 5. ([2]) Let $F$ be an unsatisfiable formula. Let $R_{1}, \ldots, R_{k}$ be a set of clauses such that

- each clause $R_{i}$ is obtained by a resolution operation where a parent clause is either a clause of $F$ or $R_{j}, j<i$;

- clauses $R_{i}$ are numbered in the derivation order and $R_{k}$ is an empty clause.

Then the $k$ resolutions that produced $R_{1}, \ldots, R_{k}$ are called a resolution proof.

\section{Boundary Points and Resolution}

The objective of this section is to discuss the relation between a resolution proof and boundary point elimination. In subsection 3.1, we define the notion of boundary points and list some of their properties. In subsection 3.2 , we recall the approach of [8] that views a resolution proof as a boundary point elimination procedure.

\subsection{Boundary points and their properties}

Definition 6. Denote by $\boldsymbol{F l s}(\boldsymbol{p}, \boldsymbol{F})$ the set of clauses of a CNF formula $F$ falsified by a complete assignment $\boldsymbol{p}$.

Definition 7. Given a CNF formula $F$, a complete assignment $\boldsymbol{p}$ is called an l-boundary point, if Fls $(\boldsymbol{p}, F) \neq \emptyset$ and every clause of $F l s(\boldsymbol{p}, F)$ contains literal $l$.

Example 1. Let $F$ consist of 5 clauses: $C_{1}=x_{2}, C_{2}=\bar{x}_{2} \vee x_{3}, C_{3}=\bar{x}_{1} \vee \bar{x}_{3}$, $C_{4}=x_{1} \vee \bar{x}_{3}, C_{5}=\bar{x}_{2} \vee \bar{x}_{3}$. Complete assignment $\boldsymbol{p}_{1}=\left(x_{1}=0, x_{2}=0, x_{3}=1\right)$ falsifies only clauses $C_{1}, C_{4}$. So $F l s\left(\boldsymbol{p}_{1}, F\right)=\left\{C_{1}, C_{4}\right\}$. There is no literal shared by all clauses of $F l s\left(\boldsymbol{p}_{\mathbf{1}}, F\right)$. Hence $\boldsymbol{p}_{\mathbf{1}}$ is not a boundary point. On the other hand, $\boldsymbol{p}_{\mathbf{2}}=\left(x_{1}=0, x_{2}=1, x_{3}=1\right)$ falsifies only clauses $C_{4}, C_{5}$ that share literal $\bar{x}_{3}$. So $\boldsymbol{p}_{\mathbf{2}}$ is a $\bar{x}_{3}$-boundary point.

Definition 8. Denote by $\boldsymbol{B n d}_{-} \boldsymbol{p n t s}(\boldsymbol{F})$ the set of all boundary points of a $C N F$ formula $F$. We assume that an l-boundary point $\boldsymbol{p}$ is specified in Bnd_pnts $(F)$ as the pair $(l, \boldsymbol{p})$. So the same point $\boldsymbol{p}$ may be present in Bnd_pnts $(F)$ more than once (e.g. if $\boldsymbol{p}$ is both an $l\left(x_{i}\right)$-boundary point and an $l\left(x_{j}\right)$-boundary point). 
Definition 9. Let $\boldsymbol{p}$ be a complete assignment. Denote by flip $\left(\boldsymbol{p}, x_{i}\right)$ the point obtained from $\boldsymbol{p}$ by flipping the value of $x_{i}$.

The following proposition explains why studying boundary points is important.

Proposition 1. ([8]) If $F$ contains at least one clause and Bnd_pnts $(F)=\emptyset$, then $F$ is unsatisfiable.

Proposition 1 implies that for a satisfiable formula $F$, Bnd_pnts $(F) \neq \emptyset$. In particular, it is not hard to show [8] that if $F\left(\boldsymbol{p}^{\prime}\right)=0, F\left(\boldsymbol{p}^{\prime \prime}\right)=1$ and $\boldsymbol{p}^{\prime \prime}=f i p\left(\boldsymbol{p}^{\prime}, x_{i}\right)$, then $\boldsymbol{p}^{\prime}$ is an $l\left(x_{i}\right)$-boundary point. (This explains the name "boundary point".) Another interesting fact is that if $\boldsymbol{p}^{\prime}$ is an $x_{i}$-boundary point, then $\boldsymbol{p}^{\prime \prime}=$ fip $\left(\boldsymbol{p}^{\prime}, x_{i}\right)$ is either a satisfying assignment or a $\bar{x}_{i}$-boundary point [8]. So, for an unsatisfiable formula, all boundary points come in pairs. We will refer to such a pair of points $\boldsymbol{p}^{\prime}$ and $\boldsymbol{p}^{\prime \prime}$ as symmetric $x_{i}$-boundary and $\bar{x}_{i}$-boundary points.

\subsection{Elimination of boundary points by adding resolvents}

Let $\boldsymbol{p}^{\prime}$ and $\boldsymbol{p}^{\prime \prime}$ be symmetric $x_{i}$-boundary and $\bar{x}_{i}$-boundary points. It is not hard to show [8] that a clause $C^{\prime}$ falsified by $\boldsymbol{p}^{\prime}$ can be resolved on variable $x_{i}$ with a clause $C^{\prime \prime}$ falsified by $\boldsymbol{p}^{\prime \prime}$. (Since points $\boldsymbol{p}^{\prime}$ and $\boldsymbol{p}^{\prime \prime}$ are $x_{i}$-boundary and $\bar{x}_{i}$-boundary respectively, $C^{\prime}$ and $C^{\prime \prime}$ have literals $x_{i}$ and $\bar{x}_{i}$ respectively. Since $\boldsymbol{p}^{\prime}, \boldsymbol{p}^{\prime \prime}$ are different only in the value of $x_{i}$, the clauses $C^{\prime}$ and $C^{\prime \prime}$ cannot have opposite literals of $x_{j}, j \neq i$.)

The resolution of $C^{\prime}$ and $C^{\prime \prime}$ above produces a clause that is falsified by both $\boldsymbol{p}^{\prime}$ and $\boldsymbol{p}^{\prime \prime}$ and does not have variable $x_{i}$. Then $\boldsymbol{p}^{\prime}$ and $\boldsymbol{p}^{\prime \prime}$ are not $l\left(x_{i}\right)$ - boundary points of $F \wedge C$. So Bnd_pnts $(F \wedge C) \subset B n d_{-} p n t s(F)$. (Adding a clause to $F$ can only eliminate some boundary points but cannot produce new ones). We will refer to this process of removing boundary points by adding clauses implied by $F$ as boundary point elimination. (Note that adding $C$ to $F$ may also eliminate a large number of $l\left(x_{i}\right)$-boundary points different from $\boldsymbol{p}^{\prime}$ and $\boldsymbol{p}^{\prime \prime}$.)

Only symmetric $x_{i}$-boundary and $\bar{x}_{i}$-boundary points can be eliminated by adding a resolvent on $x_{i}$. If formula $F$ is satisfiable, there is always an $l\left(x_{i}\right)$ boundary point $\boldsymbol{p}^{\prime}$ such that the point $\boldsymbol{p}^{\prime \prime}=f i p\left(\boldsymbol{p}^{\prime}, x_{i}\right)$ satisfies $F$ [8]. Let $C^{\prime}$ be a clause of $F$ falsified by $\boldsymbol{p}^{\prime}$ (and so having literal $l\left(x_{i}\right)$ ). The resolvent $C$ of $C^{\prime}$ with any clause $C^{\prime \prime}$ of $F$ having literal $\overline{l\left(x_{i}\right)}$ is satisfied by $\boldsymbol{p}^{\prime \prime}$ and hence by $\boldsymbol{p}^{\prime}$ (because $C$ does not depend on $x_{i}$ ). So $\boldsymbol{p}^{\prime}$ cannot be eliminated by adding a resolvent on variable $x_{i}$.

Let $R_{1}, \ldots, R_{k}$ be a resolution proof where $R_{k}$ is an empty clause. Note that Bnd_pnts $\left(F \wedge R_{k}\right)=\emptyset$. (Indeed, by definition, if $\boldsymbol{p}$ is an $l\left(x_{i}\right)$-boundary point, every clause falsified by $\boldsymbol{p}$ has to have at least one literal i.e. $l\left(x_{i}\right)$.) This means that every $l\left(x_{i}\right)$-boundary point $\boldsymbol{p}$ of the initial formula $F$ is eventually eliminated. Then there is a resolvent $R_{m+1}$ such that $\boldsymbol{p}$ is an $l\left(x_{i}\right)$-boundary point for $F \wedge R_{1} \wedge \ldots \wedge R_{m}$ but not for $F \wedge R_{1} \wedge \ldots \wedge R_{m+1}$. It was shown in [8] that $R_{m+1}$ has to be obtained by a resolution on variable $x_{i}$. In other words, an $l\left(x_{i}\right)$-boundary point mandates a resolution on variable $x_{i}$. 


\section{$4 \quad B P E-S A T$}

In this section, we describe BPE-SAT, a template SAT-solver based on the idea of Boundary Point Elimination (BPE for short).

\subsection{Justification of BPE-SAT}

BPE-SAT is based on a few very general observations we listed in the introduction. In particular, we explained why it is important to identify mandatory resolutions, i.e. ones eliminating boundary points of $F$, early. (Adding mandatory resolutions allows the SAT-solver to avoid generating many "non-mandatory" resolutions.) Finding a boundary point of $F$ is hard. If $\boldsymbol{p}$ is an $l$-boundary point it satisfies $F \backslash\{$ the clauses of $F$ with literal $l\}$. So finding a boundary point of $F$ reduces to a SAT-check for a formula that is not much smaller than $F$. BPESAT addresses this problem by using a conservative approach where boundary points are eliminated from subformulas $F^{\prime}$ of $F$. This approach is based on the following simple proposition.

Proposition 2. Let $\boldsymbol{p}$ be an l-boundary of $F$ and $F^{\prime}$ be a subset of clauses of $F$ such that $F^{\prime}(\boldsymbol{p})=0$. Then $\boldsymbol{p}$ is an l-boundary point of $F^{\prime}$.

Proof. The set of clauses of $F^{\prime}$ falsified by $\boldsymbol{p}$ is a non-empty subset of the set of clauses of $F$ falsified by $\boldsymbol{p}$.

The proposition above implies that by eliminating boundary points from $F^{\prime}$ one may also eliminate boundary points from $F$. If $F^{\prime}$ is much smaller than $F$, finding boundary points of $F^{\prime}$ is much easier than those of $F$. From the viewpoint of BPE-SAT, a subformula $F^{\prime}$ of $F$ is good if elimination of $l$-boundary points of $F^{\prime}$ also eliminates $l$-boundary points of $F$. Intuitively, finding such subformulas requires the knowledge of the structure of $F$. So, BPE-SAT does not specify how subformulas are chosen, leaving this work to implementations aimed at particular classes of formulas.

\subsection{High-level view of BPE-SAT}

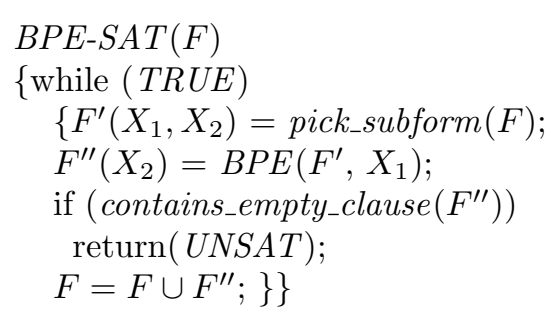

Fig. 1. $B P E-S A T$
The pseudocode of BPE-SAT is shown in Figure 1. BPE-SAT iteratively performs the following three actions. First, it picks a subformula $F^{\prime}\left(X_{1}, X_{2}\right)$ of the current formula $F$. Then it calls the BPE procedure that, for each variable $x_{i} \in X_{1}$, eliminates all symmetric $x_{i}$-boundary and $\bar{x}_{i}$-boundary points from $F^{\prime}$. If the set of clauses $F^{\prime \prime}$ generated to eliminate boundary points from $F^{\prime}$ contains an empty clause, BPE-SAT reports that $F$ is unsatisfiable. Otherwise, it adds the clauses of $F^{\prime \prime}$ to $F$ and starts a new iteration. 
In this paper, we limit the discussion of BPE-SAT to the case of unsatisfiable formulas. (The satisfiability of $F$ can be established (a) when looking for subformula $F^{\prime}$, as it is done in CDCL SAT-solvers where $F^{\prime}$ is a set of clauses responsible for a conflict; or (b) when eliminating boundary points of $F^{\prime}$, as it is done in the implementation of BPE-SAT meant for solving narrow formulas.)

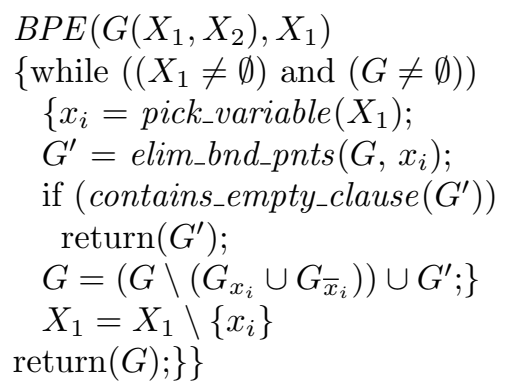

Fig. 2. $B P E$ procedure
We also do not discuss the convergence of BPE-SAT (i.e. lack of looping). Typically, the convergence of a SAT-solver with learning is achieved by avoiding the generation of the same clause more than once. An analogous approach can be used in implementations of BPE-SAT but this requires a more detailed description of the choice of subformulas $F^{\prime}$. The convergence of our implementation of BPE-SAT for narrow formulas follows from the semantics of the BPE procedure (that monotonically eliminates boundary points) and from the way subformulas $F^{\prime}$ are chosen (the choice of $F^{\prime}$ respects a fixed variable order, see Section 7).

\subsection{The BPE procedure}

The BPE procedure is described in Figure 2. It accepts a CNF formula $G\left(X_{1}, X_{2}\right)$ and processes the variables of $X_{1}$ in the "while" loop in a specified order. After a variable $x_{i}$ of $X_{1}$ is selected, elim_bnd_pnts procedure is called that eliminates all symmetric $x_{i}$-boundary and $\bar{x}_{i}$ - boundary points from $G$. It returns the set of clauses $G^{\prime}$ generated to eliminate such boundary points.

On termination of elim_bnd_pnts, $G$ is modified as follows. The clauses of $G$ with literals $x_{i}$ and $\bar{x}_{i}$ (denoted as $G_{x_{i}}$ and $G_{\bar{x}_{i}}$ respectively) are removed from $G$. The clauses $G^{\prime}$ generated by elim_bnd_pnts are added to $G$. As we show in Subsection $5,\left(G \backslash\left(G_{x_{i}} \cup G_{\bar{x}_{i}}\right)\right) \cup G^{\prime}=G\left(\ldots, x_{i}=0, \ldots\right) \vee G\left(\ldots, x_{i}=1, \ldots\right)$. That is elimination of all symmetric $x_{i}$-boundary and $\bar{x}_{i}$ - boundary points from $G$ is equivalent to quantifying away variable $x_{i}$. Finally the variable $x_{i}$ is removed from the set $X_{1}$ and a new iteration starts.

The BPE procedure terminates if 1) $G$ does not have any clauses left or 2) $X_{1}$ is empty or 3) an empty clause was derived by the elim_bnd_pnts procedure. On termination, the BPE procedure returns the current formula $G$ (that depends only on variables of $X_{2}$ due to $X_{1}=\emptyset$ ).

\subsection{Description of elim_bnd_pnts procedure}

Given a CNF formula $G$ and variable $x_{i}$, the elim_bnd_pnts procedure (shown in Figure 3) returns a set of resolvents $G^{\prime}$ eliminating all symmetric $x_{i}$-boundary and $\bar{x}_{i}$-boundary points of $G$ for $x_{i} \in X_{1}$. (That is, if an $l\left(x_{i}\right)$-boundary point $\boldsymbol{p}$ is left in $G \wedge G^{\prime}$, then $\boldsymbol{p}^{*}=f i p\left(\boldsymbol{p}, x_{i}\right)$ satisfies $G$ and so $\boldsymbol{p}$ cannot be eliminated.) 
Boundary points are generated in the "while" loop. Every time a new $l\left(x_{i}\right)$ boundary point $\boldsymbol{p}$ is found, it is eliminated by adding a resolvent on variable $x_{i}$ that is falsified by $\boldsymbol{p}$. The procedure terminates when all symmetric $x_{i}$-boundary and $\bar{x}_{i}$-boundary points are eliminated. Below, we describe elim_bnd_pnts in more detail.

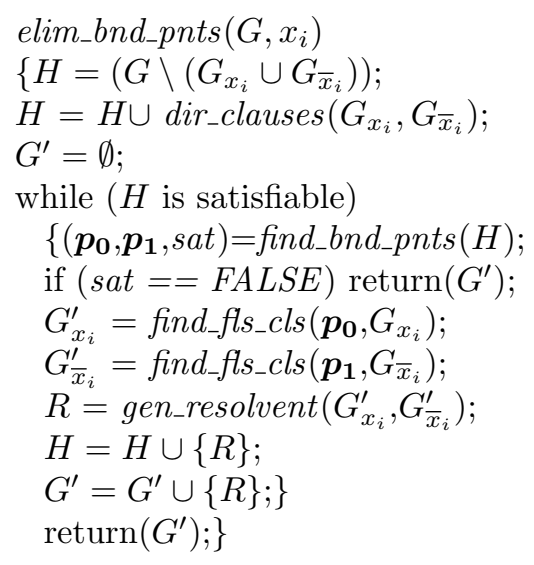

Following the definition of an $l\left(x_{i}\right)$ boundary point, elim_bnd_pnts looks for an assignment satisfying formula $H=G \backslash\left(G_{x_{i}} \cup G_{\bar{x}_{i}}\right)$. Adding directing clauses to $H$ (constructed by function dir_clauses) is meant to avoid generation of $l\left(x_{i}\right)$-boundary points that cannot be eliminated. This is achieved by avoiding assignments satisfying $G_{x_{i}}$ and/or $G_{\bar{x}_{i}}$. A more detailed description of generation of directing clauses is given at the bottom of this subsection.)

The find_bnd_pnts procedure called in the "while" loop is a CDCL SATsolver. It returns a pair of assignments $\boldsymbol{p}_{\mathbf{0}}, \boldsymbol{p}_{\mathbf{1}}$ symmetric in $x_{i}$ that satisfy $H$ (by construction, $H$ does not depend on

Fig. 3. elim_bnd_pnts procedure $x_{i}$ ) or reports that $H$ is unsatisfiable. For the sake of clarity, we assume that $x_{i}=0$ (respectively $x_{i}=1$ ) in $\boldsymbol{p}_{\mathbf{0}}$ (respectively $\boldsymbol{p}_{\mathbf{1}}$ ). Then $\boldsymbol{p}_{\mathbf{0}}, \boldsymbol{p}_{\mathbf{1}}$ are $x_{i}$-boundary and $\bar{x}_{i}$-boundary points of $G$ respectively.

If $H$ is unsatisfiable, all symmetric $x_{i}$-boundary and $\bar{x}_{i}$-boundary points have been eliminated and elim_bnd_pnts terminates returning the set $G^{\prime}$ of generated resolvents. Otherwise, the clauses $G_{x_{i}}^{\prime} \subseteq G_{x_{i}}$ (respectively $G_{\bar{x}_{i}}^{\prime} \subseteq G_{\bar{x}_{i}}$ ) are generated that are falsified by $\boldsymbol{p}_{\mathbf{0}}$ (respectively $\boldsymbol{p}_{\mathbf{1}}$ ). Note that due to adding to $H$ directing clauses, the sets $G_{x_{i}}^{\prime}$ and $G_{\bar{x}_{i}}^{\prime}$ cannot be empty. Boundary points $\boldsymbol{p}_{\mathbf{0}}$ and $\boldsymbol{p}_{1}$ can be eliminated by resolving any clause of $G_{x_{i}}^{\prime}$ with any clause of $G_{\bar{x}_{i}}^{\prime}$.

The choice of the resolvent eliminating boundary points $\boldsymbol{p}_{\mathbf{0}}$ and $\boldsymbol{p}_{\mathbf{1}}$ is done by the procedure gen_resolvent. The latter uses a heuristic illustrated by the following example. Suppose boundary points $\boldsymbol{p}_{\mathbf{0}}$ and $\boldsymbol{p}_{\mathbf{1}}$ are found by the BPE procedure when processing variable $x_{5}$. Let $R^{\prime}=x_{10} \vee x_{20}$ and $R^{\prime \prime}=x_{20} \vee \bar{x}_{35} \vee x_{40}$ be the two clauses obtained by resolving clauses of $G_{x_{5}}^{\prime}$ and $G_{\bar{x}_{5}}^{\prime}$ (and so eliminating $\boldsymbol{p}_{\mathbf{0}}$ and $\boldsymbol{p}_{\mathbf{1}}$ as $l\left(x_{5}\right)$-boundary points). Given a clause $C$, denote by min_index $(C)$ the minimum variable index among $\operatorname{Vars}(C)$. Hence min_index $\left(R^{\prime}\right)=10$ and min_index $\left(R^{\prime \prime}\right)=20$. The gen_resolvent procedure selects the clause with the largest value of min_index (in our case it is the clause $R^{\prime \prime}$ ).

The reason for using the heuristic above is as follows. In our implementation of the BPE procedure, variables $x_{i}$ are processed in the order they are numbered. Let clause $R^{\prime}$ be chosen to eliminate $\boldsymbol{p}_{\mathbf{0}}$ and $\boldsymbol{p}_{\mathbf{1}}$. Suppose that after elimination of all symmetric $x_{5}$-boundary and $\bar{x}_{5}$-boundary points and removing the clauses with variable $x_{5}$ from $G$ (see Figure 2), $R^{\prime}$ is the only clause of $G$ falsified 
by $\boldsymbol{p}_{\mathbf{0}}$ and $\boldsymbol{p}_{\mathbf{1}}$. Then, say, $\boldsymbol{p}_{\mathbf{0}}$ is an $x_{10}$-boundary point of $G$. This means that when processing variable $x_{10}$, the point $\boldsymbol{p}_{\mathbf{0}}$ and the point flip $\left(\boldsymbol{p}_{\mathbf{0}}, x_{10}\right)$ need to be eliminated (and hence may cause an extra run of the SAT-solver used for finding $l\left(x_{10}\right)$-boundary points.) On the other hand, if $R^{\prime \prime}$ is chosen to eliminate $\boldsymbol{p}_{\mathbf{0}}$ and $\boldsymbol{p}_{\mathbf{1}}$, no extra work is needed until variable $x_{20}$ is processed. In other words, when eliminating $l\left(x_{i}\right)$-boundary points $\boldsymbol{p}_{\mathbf{0}}$ and $\boldsymbol{p}_{\mathbf{1}}$, gen_resolvent tries to minimize the number of $l\left(x_{j}\right)$-boundary points the points $\boldsymbol{p}_{\mathbf{0}}$ and $\boldsymbol{p}_{\mathbf{1}}$ turn into (where $i<j$ ) after removing the clauses with variable $x_{i}$.

Avoiding the generation of boundary points that cannot be eliminated is done by find_bnd_pnts as follows. Let $\boldsymbol{q}$ be a partial assignment such that $G_{x_{i}}(\boldsymbol{q})=1$ and/or $G_{\bar{x}_{i}}(\boldsymbol{q})=1$. Note that no boundary point $\boldsymbol{p}$ obtained by extension of $\boldsymbol{q}$ can be eliminated by a resolvent of a clause of $G_{x_{i}}$ with a clause of $G_{\bar{x}_{i}}$ (because any such a resolvent is satisfied by $\boldsymbol{p}$ ). To make find_bnd_pnts avoid the regions of the search space where $G_{x_{i}}$ and/or $G_{\bar{x}_{i}}$ are satisfied, new clauses are added to $H$ by the dir_clauses procedure.

Now we explain how avoiding the regions where $G_{x_{i}}=1$ is done. (The new clauses meant to avoid regions where $G_{\bar{x}_{i}}=1$ are built analogously.) First, a new variable $y_{c}$ is introduced for each clause of $C$ of $G_{x_{i}}$. Then the clauses describing the equality $y_{c} \equiv C^{\prime}$ are added to $H$ where $C^{\prime}$ is $C$ minus $x_{i}$. To guarantee that at least one clause of $G_{x_{i}}$ stays falsified when $x_{i}=0$, the clause consisting of the literals $\overline{y_{c}}$ is added to $H$.

\section{Quantification by Boundary Point Elimination}

In this section, we show that elimination of all symmetric $x_{i}$-boundary and $\bar{x}_{i}$ boundary points of a CNF formula $G$ is equivalent to existentially quantifying away variable $x_{i}$ from $G$. This means that the BPE procedure simply quantifies away the variables of $X_{1}$ (see Figure 2). This result shows that the BPE approach can be used for existential quantification. (And as we argue in Section 6, existential quantification is one of the bottlenecks of CDCL SAT-solvers). At the same time, we believe that the naive algorithm of the BPE procedure, where boundary points are eliminated variable by variable, can be significantly improved.

Proposition 3. Let $G\left(x_{1}, \ldots, x_{n}\right)$ be a CNF formula. Let $G_{x_{i}}$ (respectively $G_{\bar{x}_{i}}$ ) be the set of clauses of $G$ having literal $x_{i}$ (respectively literal $\bar{x}_{i}$ ). Let $G^{\prime}$ be a set of resolvents where, for every resolvent, one parent clause is from $G_{x_{i}}$ and the other is from $G_{\bar{x}_{i}}$. Let $G \wedge G^{\prime}$ do not have any pair of symmetric $x_{i}$-boundary and $\bar{x}_{i}$-boundary points. Then

$G\left(\ldots, x_{i}=0, \ldots\right) \vee G\left(\ldots, x_{i}=1, \ldots\right)=\left(G \backslash\left(G_{x_{i}} \cup G_{\bar{x}_{i}}\right)\right) \cup G^{\prime}$.

Proof. See Appendix A.

Proposition 3 implies that the BPE procedure is complete. Namely, by eliminating the symmetric $x_{i}$-boundary and $\bar{x}_{i}$-boundary points for all variables of $G$, the BPE procedure existentially quantifies away all variables of $G$. This results either in producing an empty set of clauses (which means that $G$ is satisfiable) or in generating an empty clause ( $G$ is unsatisfiable). 


\section{CDCL SAT-solvers in terms of BPE}

In this section, we give a brief analysis of CDCL SAT-solvers in BPE terms. On the one hand, a CDCL SAT-solver is a particular implementation of BPESAT. (This is very interesting taking into account that BPE-SAT is a theoretical construction based on a few general observations.) On the other hand, the BPE approach exposes two problems of CDCL SAT-solvers that, we believe, can be addressed in more advanced implementations of BPE-SAT. These problems are important because their solution will enable structure-aware SAT-solvers.

A CDCL SAT-solver is an implementation of BPE-SAT. A key part of a CDCL SAT-solver is its conflict clause generation procedure [10,13]. Let $F^{\prime}\left(X_{1}, X_{2}\right)$ be the set of clauses responsible for a conflict. Here $X_{1}$ is the set of variables in which clauses of $F^{\prime}$ have literals of both polarities and $X_{2}=$ $\operatorname{Vars}\left(F^{\prime}\right) \backslash X_{1}$. A conflict clause generation procedure is, essentially, the DP procedure [6] that resolves out the variables of $X_{1}$ from $F^{\prime}$ one by one eventually producing a conflict clause $C$ (where $\operatorname{Vars}(C)=X_{2}$ ). So, a CDCL SAT-solver is very similar to an implementation of BPE-SAT where subformulas $F^{\prime}$ are subsets of clauses responsible for conflicts. However, the similarity goes much deeper. It can be shown that a conflict clause generation procedure is essentially a special case of the BPE-procedure that produces resolvents eliminating boundary points of subformula $F^{\prime}$. Interestingly, all the required boundary points can be trivially obtained from the partial assignment leading to the conflict specified by $F^{\prime}$. (The details are given is Appendix B.) So, a CDCL SAT-solver is an implementation of BPE-SAT.

Eager backtracking interferes with finding subformulas $F^{\prime}$ sharing boundary points with $F$. Let $F^{\prime}\left(X_{1}, X_{2}\right)$ be the set of clauses responsible for a conflict produced by a CDCL SAT-solver in CNF formula $F$. Let $A$ and $B$ be clauses of $F^{\prime}$ resolved on variable $x_{i} \in X_{1}$ when generating the conflict clause. Let $\boldsymbol{q}$ be an assignment to $\operatorname{Vars}\left(F^{\prime}\right)$ such that $\boldsymbol{q}$ and $\boldsymbol{q}^{*}=\operatorname{fip}\left(\boldsymbol{q}, x_{i}\right)$ are symmetric $x_{i}$-boundary and $\bar{x}_{i}$-boundary points of $F^{\prime}$. (Although it is not crucial for our reasoning here, the existence of $\boldsymbol{q}$ can actually be proved similarly to Appendix B.)

Importantly, no guarantees can be made that an $l\left(x_{i}\right)$-boundary of the entire current formula $F$ is eliminated by adding the resolvent of $A$ and $B$. For example, it may be the case that no matter how $\boldsymbol{q}$ is extended by assignments to the variables of $\operatorname{Vars}\left(F \backslash F^{\prime}\right)$, the extended assignment falsifies a clause of $F \backslash F^{\prime}$ that does not have variable $x_{i}$ (and so this extended assignment cannot be an $l\left(x_{i}\right)$-boundary point of $\left.F\right)$. As we show in Section 9 , when a SAT-solver only "accidentally" runs into conflicts that lead to generation of resolutions eliminating boundary points of $F$, its performance may be very poor. One can address this problem by allowing a SAT-solver to make decision assignments after a conflict occurs. (This may lead to finding another conflict specifying a much better subformula $F^{\prime}$.) However, it is not clear if this can be done efficiently: eager backtracking is a cornerstone of CDCL SAT-solvers.

$C D C L$ SAT-solvers and existential quantification. BPE-SAT works with a more general class of subformulas $F^{\prime}$ (whose variables are existentially quantified away) than CDCL SAT-solvers. A natural question is if this is beneficial 
for BPE-SAT. According to [9], given a resolution proof $R$ that a CNF formula $F$ is unsatisfiable, a non-deterministic CDCL SAT-solver can build another resolution proof that may be larger than $R$ only by $|\operatorname{Vars}(F)|^{4}$. Since BPE-SAT and CDCL SAT-solvers are based on resolution it is tempting to conclude that a deterministic CDCL SAT-solver can efficiently simulate a proof generated by an implementation of BPE-SAT. However, this conclusion may turn out to be wrong because the termination conditions of deterministic and non-deterministic CDCL SAT-solvers are different when performing existential quantification.

Suppose that a non-deterministic CDCL SAT-solver performs existential quantification of $F^{\prime}\left(X_{1}, X_{2}\right)$ by generating clauses depending on $X_{2}$ and proving that they are implied by $F^{\prime}$. Such a SAT-solver stops as soon as a set of clauses $F^{\prime \prime}\left(X_{2}\right)$ forming a solution is generated. On the contrary, a deterministic CDCL SAT-solver, even if $F^{\prime \prime}\left(X_{2}\right)$ is generated has to show that any assignment to $X_{2}$ satisfying $F^{\prime \prime}$ can be extended (by assignments to variables of $X_{1}$ ) to an assignment satisfying $F^{\prime}$. (I.e. such a SAT-solver has to prove that $F^{\prime \prime}$ is indeed a solution). This may require some extra work that can be even exponential!. The observation above implies that developing efficient methods of existential quantification is very important for creation of new powerful SAT-solvers.

\section{Applying BPE-SAT to Solving Narrow Formulas}

In this section, we describe an implementation of BPE-SAT meant for solving narrow formulas. We picked this class of formulas for the following reasons. First, narrow formulas occur in practical applications. Second, the choice of good subformulas for narrow formulas is very simple.

Let $F$ be a CNF formula. Let $\operatorname{Vars}(F)=\left\{x_{1}, \ldots, x_{n}\right\}$. Denote by $\prec$ the order on $\operatorname{Vars}(F)$ specified by variable numbering (i.e. $\left(x_{i} \prec x_{j}\right) \equiv(i<j)$ ). Denote by $F^{i}$ the subset of clauses of $F$ that have variable $x_{j}, j \leq i$. Denote by $F^{i *}$ the set of clauses $F \backslash F^{i}$. The value of $\left|\operatorname{Vars}\left(F^{i}\right) \cap \operatorname{Vars}\left(F^{i *}\right)\right|$ (denoted as $\left.w\left(F, x_{i}, \prec\right)\right)$ is called the width of formula $F$ at variable $x_{i}$ with respect to order $\prec$. The maximum value among $w\left(F, x_{i}, \prec\right), i=1, \ldots, n$ (denoted as $\left.w(F, \prec)\right)$ is called the width of $F$ with respect to order $\prec$. We assume in this section that the order $\prec$ specifies a minimum (or close to minimum) width with respect to all possible orders on $X$.

Informally, a CNF formula $F$ has a small width if $w(F, \prec) \ll|\operatorname{Vars}(F)|$. To apply BPE-SAT to $F$ we use $F^{i}$ as a subformula $F^{\prime}\left(X_{1}, X_{2}\right)$ of $F$. (Here $X_{1}=\left\{x_{1}, \ldots, x_{i}\right\}$ and $X_{2}=\operatorname{Vars}(F) \backslash X_{1}$.) The advantage of such a choice is twofold. First, since $F$ is narrow, if $i$ is small then $F^{\prime}$ is also small. (In our experiments the value of $i$ was set to 100). So finding boundary points of $F^{\prime}$ is easy. Second, any $l\left(x_{i}\right)$-boundary point of $F$ (where $\left.x_{i} \in X_{1}\right)$ is also an $l\left(x_{i}\right)$ boundary point of $F^{\prime}$ (because the latter contains all clauses of $F$ with $x_{i}$ ). So $F^{\prime}$ satisfies the criterion of good subformulas of Subsection 4.1.

After quantifying away the variables of $X_{1}$ from $F^{\prime}$, a set of clauses $F^{\prime \prime}\left(X_{2}\right)$ is generated. The clauses with variables of $X_{1}$ are removed from $F$ and the clauses of $F^{\prime \prime}$ are added to $F$. (Note that in the pseudocode of BPE-SAT described in 
Figure 1 we do not remove clauses with variables of $X_{1}$ from $F$. We can do these for narrow formulas because, initially, $F^{\prime}$ contains all the clauses of $F$ with variables of $X_{1}$.) Then the same procedure is applied to $F$ again. That is $F^{\prime}$ is the set of clauses of $F$ with a variable $x_{j}, j \leq 2 * i$. (Here we take into account the fact that variables $x_{1}, \ldots, x_{i}$ have been removed from $F$.) Eventually, either an empty clause is derived (the original formula $F$ is unsatisfiable) or all clauses are removed from $F$ (the original formula $F$ is satisfiable).

\section{Some Background}

SAT-solving of real-life CNF formulas has been dominated by algorithms based on the DPLL procedure [5] (e.g. [10,11]). Recently, considerable effort has been given to understanding the reasons for success of CDCL SAT-solvers. In

Table 1. Solving formulas describing equivalence checking of identical copies of $n$-bit multipliers

\begin{tabular}{|c|c|c|c|c|}
\hline \#bits & $\begin{array}{c}\text { \#vars, } \\
\# \text { cls }) \\
\times 10^{3}\end{array}$ & $\begin{array}{c}\text { Picosat } \\
\# \text { res. } \\
\times 10^{6}\end{array}$ & $\begin{array}{c}\text { Spec. } \\
\text { \#res. } \\
\times 10^{3}\end{array}$ & $\begin{array}{c}\text { Proof } \\
\text { size } \\
\text { ratio }\end{array}$ \\
\hline 6 & $(0.5,1.6)$ & 1.2 & 1.9 & 631 \\
\hline 7 & $(0.7,2.2)$ & 7.3 & 2.7 & 2,703 \\
\hline 8 & $(1.0,3.0)$ & 45 & 3.6 & 12,500 \\
\hline 9 & $(1.2,3.8)$ & 249 & 4.7 & 52,978 \\
\hline
\end{tabular}

[12] (and the follow-up papers) properties of real-life formulas have been studied. In particular, it was conjectured that such formulas may have very small "backdoors" i.e. sets of variables assigning which dramatically reduces formula complexity. In a number of papers (e.g. [9]), the success of CDCL SAT-solvers has been related to the ability of their non-deterministic counterparts to simulate resolution proofs.

In [8], we showed that a resolution proof can be viewed as a process of boundary point elimination. This result allows one to draw some conclusions about properties of deterministic SAT-solvers based on resolution. In particular, the existence of mandatory resolutions (that require explicitly or implicitly looking for boundary points) sheds some light on why SAT-solvers based on the DPLL procedure dominate among the resolution based algorithms.

\section{Experimental Results}

The goal of experiments was twofold. First, we wanted to relate poor performance of CDCL SAT-solvers on some formulas with non-trivial structure to their inability to identify (and hence eliminate) boundary points. Second, we tested a very simple implementation of BPE-SAT tailored to narrow formulas and compared it with CDCL SAT-solvers. Narrow formulas $F(r, m)$ we used in experiments can be relatively easily solved using BDDs. However, neither BPE-SAT nor CDCL SAT-solvers use subformula hashing employed by BDDs. Importantly, it is not clear how one could use the structure of these formulas in a CDCL SAT-solver. For example, our implementation of BPE-SAT can successfully solve formulas $F(4, m)$ for a variable ordering for which the formula width is 92 . This makes the trivial algorithm of quantifying away the variables of $X_{1}$ 
from subformula $F^{\prime}\left(X_{1}, X_{2}\right)$ (where one just enumerates assignments to $X_{2}$ ) inefficient. In Appendix C, we also show experimentally that the DP procedure (that is operationally similar to the BPE procedure of Figure 2) is dramatically slower than BPE-SAT on formulas $F(r, m)$. The experiments were run on a Linux machine with Intel Core 2 Duo CPU with $3.16 \mathrm{GHz}$ clock frequency.

Table 1 compares the size of resolution

Table 2. Comparison of Picosat proofs with specialized ones

\begin{tabular}{|c|c|c|c|c|}
\hline \#bits & \multicolumn{2}{|c|}{ Picosat } & \multicolumn{2}{c|}{ Specialized } \\
\cline { 2 - 5 } & \#res. & $\begin{array}{l}\text { mand. } \\
\text { res. \% }\end{array}$ & \#res. & $\begin{array}{l}\text { mand. } \\
\text { res. \% }\end{array}$ \\
\hline 2 & 215 & 77 & 77 & 100 \\
\hline 3 & 2,958 & 66 & 409 & 100 \\
\hline 4 & 32,117 & 38 & 957 & 100 \\
\hline 5 & 231,270 & 24 & 1,697 & 100 \\
\hline
\end{tabular}
proofs for CNF formulas describing equivalence checking of two identical copies of $n$-bit multipliers. The first column gives the value of $n$ for the multipliers checked for equivalence. The second column shows the number of variables and clauses (in thousands). The next column gives the number of resolutions in the proofs generated by the SAT-solver Picosat [4] (in millions). (Picosat is the version of Precosat, a winner of the SAT-2009 competition, that does not use formula preprocessing. Since Precosat does not generate proofs, we used Picosat instead). The size of specialized proofs that take into account the formula structure [8] (in thousands of resolutions) is given in the fourth column. The ratio of the sizes of Picosat proofs and specialized ones is shown in the last column.

The results of Table 1 show

Table 3. Solving formulas $F(r, m)$. Time limit is $3 \times 10^{5}$ seconds. Symbol ' $*$ ' marks the timeouts.

\begin{tabular}{|l|l|l|l|l|l|l|}
\hline$(r, m)$ & $\begin{array}{l}(\# \text { vars, } \\
\# c l s) \\
\times 10^{3}\end{array}$ & $\begin{array}{l}\text { wi- } \\
\text { dth }\end{array}$ & $\begin{array}{l}\text { Mini- } \\
\text { sat } \\
(\mathrm{s} .)\end{array}$ & $\begin{array}{l}\text { Pico- } \\
\text { sat } \\
(\mathrm{s} .)\end{array}$ & $\begin{array}{l}\text { BPE } \\
(\mathrm{s} .)\end{array}$ & $\begin{array}{l}\text { ratio } \\
\text { Pico/ } \\
\text { BPE }\end{array}$ \\
\hline$(2,800)$ & $(10,27)$ & 8 & 0.2 & 0.5 & 3.3 & 0.2 \\
\hline$(2,1300)$ & $(17,44)$ & 8 & 0.7 & 0.7 & 6.6 & 0.1 \\
\hline$(2,3000)$ & $(39,102)$ & 8 & 6.0 & 3.4 & 25.3 & 0.1 \\
\hline \hline$(3,300)$ & $(11,30)$ & 16 & 18.9 & 2.3 & 6.4 & 0.4 \\
\hline$(3,1000)$ & $(35,100)$ & 16 & 536 & 69.6 & 30.8 & 2.2 \\
\hline$(3,2000)$ & $(70,200)$ & 16 & 6,456 & 162 & 88.0 & 1.8 \\
\hline \hline$(4,200)$ & $(29,86)$ & 29 & 2,099 & 548 & 58.7 & 9.3 \\
\hline$(4,600)$ & $(88,258)$ & 29 & $*$ & 99,820 & 235 & 425 \\
\hline$(4,1000)$ & $(147,430)$ & 29 & $*$ & $*$ & 955 & $>302$ \\
\hline
\end{tabular}
that in comparison to specialized proofs, Picosat proofs are very large. We also tried other well known SAT-solvers (that do not generate proofs) like Minisat, Precosat and Glucose. In terms of the number of backtracks their performance was similar to $\mathrm{Pi}$ cosat's.

To explain the difference in proof quality, we computed the value of the SMR metric [8] for the Picosat proofs and the specialized ones. The results are given in Table 2. Given a resolution proof $R$ that a CNF formula $F$ is unsatisfiable, the value of SMR-metric is the Share of Mandatory Resolutions in $R$ i.e. share of resolutions eliminating boundary points not eliminated by previous resolutions. (In [8] this metric was called Share of Boundary Resolutions).

In Table 2 we used the same kind of formulas as in Table 1 but of smaller size (computation of SMR metric is expensive.) Table 2 shows that the value of SMR metric for specialized proofs remains $100 \%$. (That is these proofs consist 
entirely of mandatory resolutions.) On the contrary, the value of SMR metric for Picosat's proofs sharply decreases. This can be attributed to the poor choice of subformulas by CDCL SAT-solvers (see Section 6).

Table 3 describes the experiment with

Table 4. Proofs generated by Picosat and BPE-SAT for narrow formulas

\begin{tabular}{|c|c|c|c|c|}
\hline$(r, m)$ & $\begin{array}{c}(\# \text { vars, } \\
\text { \#cls }) \\
\times 10^{3}\end{array}$ & $\begin{array}{c}\text { Picosat } \\
\text { \#res. } \\
\times 10^{3}\end{array}$ & $\begin{array}{c}\text { BPE } \\
\text { \#res. } \\
\times 10^{3}\end{array}$ & $\begin{array}{c}\text { Proof } \\
\text { size } \\
\text { ratio }\end{array}$ \\
\hline$(3,10)$ & $(0.4,1.0)$ & 23.4 & 2.6 & 9.0 \\
\hline$(3,20)$ & $(0.7,2.0)$ & 96.6 & 5.3 & 18.2 \\
\hline$(3,30)$ & $(1.1,3.0)$ & 123 & 8.0 & 15.4 \\
\hline$(3,40)$ & $(1.4,4.0)$ & 206 & 10.7 & 19.3 \\
\hline$(4,10)$ & $(1.4,4.0)$ & 256 & 19.3 & 13.3 \\
\hline$(4,20)$ & $(2.8,8.2)$ & 856 & 35.4 & 24.2 \\
\hline$(4,30)$ & $(4.3,12.5)$ & 3,415 & 51.6 & 66.2 \\
\hline$(4,40)$ & $(5.7,16.9)$ & 3,036 & 67.7 & 44.8 \\
\hline
\end{tabular}

and $N^{\prime}$ had $m+r-1$ inputs.

For solving formulas $F(r, m)$ we used an implementation of BPE-SAT tailored to narrow formulas. Subformulas $F^{\prime}$ of $F(r, m)$ were chosen as described in Section 7. For finding boundary points we used the same version of Picosat that was employed in other experiments of this section.

In Table 3, we compare BPE-SAT with Minisat (versolving narrow formulas. We used a class of unsatisfiable formulas $F(r, m), r>1$, $m>0$ specifying equivalence checking of narrow circuits [3]. (Equivalence checking was employed here just as a simple way to produce unsatisfiable formulas. The formulas we used in experiments and their description can be downloaded from [14]). The two circuits $N$ and $N^{\prime}$ we checked for equivalence had the same high-level structure specified by parameters $r$ and $m$. Circuits $N$ and $N^{\prime}$ mimicked the structure of an $m$-bit adder with $r$ carry bits. So $N$ and $N^{\prime}$ consisted of a cascade of $m$ blocks communicating with each other by $r$ wires. $N$
Table 5. SMR metric for proofs generated by Picosat and BPE-SAT

\begin{tabular}{|c|c|c|}
\hline$(r, m)$ & $\begin{array}{c}\text { Picosat } \\
\text { mand. } \\
\text { res. \% }\end{array}$ & $\begin{array}{c}\text { BPE } \\
\text { mand. } \\
\text { res. \% }\end{array}$ \\
\hline$(3,10)$ & 18.4 & 86.5 \\
\hline$(3,20)$ & 10.6 & 90.3 \\
\hline$(3,30)$ & 16.5 & 92.1 \\
\hline$(3,40)$ & 13.7 & 92.9 \\
\hline$(4,10)$ & 17.2 & 60.7 \\
\hline$(4,20)$ & 12.2 & 75.5 \\
\hline$(4,30)$ & 7.5 & 80.6 \\
\hline$(4,40)$ & 7.7 & 83.5 \\
\hline
\end{tabular}
sion 2.0) and Picosat on formulas $F(r, m), r \in\{2,3,4\}$. The first column gives the value of parameters $r$ and $m$. The third column specifies the value $w(F, \prec)$ of the formula width (defined in Section 7 ). The next three columns give the performance of Minisat, Picosat and BPE-SAT in seconds. The last column shows the ratio of Picosat's and BPE-SAT's runtimes.

The formulas $F(2, m)$ are easily solved by each SATsolver, with Picosat having the best performance. The formulas $F(3, m)$ of Table 3 are hard for Minisat for $m=2000$. Picosat is competitive with BPE-SAT and scales up well. This changes for formulas $F(4, m)$. For example, when the formula size increases 3 times (from $F(4,200)$ to $F(4,600))$ the runtime of Picosat increases 182 times (from 548 to 99,820 seconds).

In Tables 4 and 5, we analyze proofs generated by Picosat and BPE-SAT for narrow formulas. Table 4 shows the size of proofs generated by Picosat and BPE-SAT (in thousands of resolutions). The proofs of Picosat are up to 66 times larger than those of BPE-SAT even for small values of $m$. (For greater values of $m$, the proofs generated by Picosat were too large to 
process.) Table 5 sheds some light on why proof sizes are different: the value of SMR metric for the proofs of Picosat is much lower than for those of BPE-SAT.

\section{Conclusions}

The fact that the resolution proof system is most likely non-automatizable implies that a resolution-based SAT-solver, in general, needs to know the formula structure to be successful. In this paper, we use the Boundary Point Elimination (BPE) concept to study the problem of building structure-aware SATsolvers. We show that although the behavior of CDCL SAT-solvers from the viewpoint of BPE is quite reasonable, they have at least two flaws. First, eager backtracking of a CDCL SAT-solver makes it hard to generate resolutions that eliminate boundary points of the formula. Second, a CDCL SAT-solver cannot efficiently perform existential quantification. We introduce a template of resolution SAT-solvers called BPE-SAT meant for addressing these problems and hence for building structure-aware SAT-algorithms.

\section{References}

1. M. Alekhnovich and A. Razborov. Resolution is not automatizable unless $\mathrm{w}[\mathrm{p}]$ is tractable. SIAM J. Comput., 38(4):1347-1363, 2008.

2. L. Bachmair and H. Ganzinger. Resolution theorem proving. In A. Robinson and A. Voronkov, editors, Handbook of Automated Reasoning, volume I, chapter 2, pages 19-99. North-Holland, 2001.

3. L. Berman. Circuit width, register allocation, and ordered binary decision diagrams. IEEE Trans. on CAD of Integr. Circ. and Syst., 10(8):1059-1066, 1991.

4. A. Biere. Picosat essentials. JSAT, 4(2-4):75-97, 2008.

5. M. Davis, G. Logemann, and D. Loveland. A machine program for theorem proving. Communications of the ACM, 5(7):394-397, July 1962.

6. M. Davis and H. Putnam. A computing procedure for quantification theory. Journal of the ACM, 7(3):201-215, July 1960.

7. R. Dechter and I. Rish. Directional resolution: The davis-putnam procedure, revisited. In KR, pages 134-145, 1994.

8. E. Goldberg. Boundary points and resolution. In SAT-09, pages 147-160, Berlin, Heidelberg, 2009. Springer-Verlag.

9. K.Pipatsrisawat and A.Darwiche. On the power of clause-learning SAT solvers with restarts. In Proceedings of CP-2009, pages 654-668, September 2009.

10. J. Marques-Silva and K. Sakallah. Grasp - a new search algorithm for satisfiability. In ICCAD-96, pages 220-227, Washington, DC, USA, 1996.

11. M. Moskewicz, C. Madigan, Y. Zhao, L. Zhang, and S. Malik. Chaff: engineering an efficient sat solver. In $D A C-01$, pages 530-535, New York, NY, USA, 2001.

12. R. Williams, C. P. Gomes, and B. Selman. Backdoors to typical case complexity. In IJCAI, pages 1173-1178, 2003.

13. L. Zhang and S. Malik. Conflict driven learning in a quantified boolean satisfiability solver. In ICCAD, pages 442-449, 2002.

14. http://eigold.tripod.com/benchmarks/narrow_formulas.tar.gz. 


\section{Appendix}

\section{A Proof of Proposition 3}

Proof. Denote by $H_{l}$ and $H_{r}$ the left and right parts of the equality to be proved i.e. $G\left(\ldots, x_{i}=0, \ldots\right) \vee G\left(\ldots, x_{i}=1, \ldots\right)$ and $\left(G \backslash\left(G_{x_{i}} \cup G_{\bar{x}_{i}}\right)\right) \cup G^{\prime}$ respectively.

1) Proving $H_{l}=1 \rightarrow H_{r}=1$. Let $\boldsymbol{p}$ be an assignment to the variables of $H_{l}$ for which $H_{l}(\boldsymbol{p})=1$. By definition of $H_{l}$, there is an assignment to $x_{i}$ such that by adding it to $\boldsymbol{p}$ one obtains an assignment $\boldsymbol{p}^{*}$ satisfying $G$. Every clause of $H_{r}$ is either a clause of $G$ or a resolvent of clauses of $G$. So $H_{r}\left(\boldsymbol{p}^{*}\right)=1$. Since $H_{r}$ does not depend on $x_{i}, H_{r}(\boldsymbol{p})=1$ too.

2) Proving $H_{l}=0 \rightarrow H_{r}=0$. Let $\boldsymbol{p}$ be an assignment to the variables of $H_{l}$ for which $H_{l}(\boldsymbol{p})=0$. Denote by $\boldsymbol{p}_{\mathbf{0}}$ and $\boldsymbol{p}_{\mathbf{1}}$ the assignments obtained from $\boldsymbol{p}$ by adding assignments $x_{i}=0$ and $x_{i}=1$ respectively. By definition of $H_{l}, G\left(\boldsymbol{p}_{\mathbf{0}}\right)=$ $G\left(\boldsymbol{p}_{\mathbf{1}}\right)=0$. Let us consider the following two cases.

a) Points $\boldsymbol{p}_{\mathbf{0}}$ and $\boldsymbol{p}_{\mathbf{1}}$ falsify a clause $C$ of $G$ that does not have variable $x_{i}$. Then $C$ is in $H_{r}$ and so $H_{r}(\boldsymbol{p})=0$.

b) Points $\boldsymbol{p}_{\mathbf{0}}$ and $\boldsymbol{p}_{\mathbf{1}}$ falsify only clauses of $G$ with variable $x_{i}$. This means that $\boldsymbol{p}_{\mathbf{0}}$ and $\boldsymbol{p}_{\mathbf{1}}$ falsify at least one clause of $G_{x_{i}}$ and $G_{\bar{x}_{i}}$ respectively. Then $\boldsymbol{p}_{\mathbf{0}}$ and $\boldsymbol{p}_{\mathbf{1}}$ are symmetric $x_{i}$-boundary and $\bar{x}_{i}$-boundary points. By definition, $G \wedge G^{\prime}$ does not have such a pair of points. So $G^{\prime}$ contains a resolvent on $x_{i}$ that is falsified by $\boldsymbol{p}_{\mathbf{0}}$ and $\boldsymbol{p}_{\mathbf{1}}$. Since this resolvent is in $H_{r}, H_{r}(\boldsymbol{p})=0$.

\section{B CDCL SAT-solvers are a special case of BPE-SAT}

In this section, we show that the conflict clause generation procedure [13] of a CDCL-SAT-solver is a special case of the BPE procedure and hence a CDCL SAT-solver is a special case of BPE-SAT. We assume that the reader is familiar with the basic notions of CDCL SAT-solvers [10,11]. First, we describe a simple example and then give a formal description.

Example 2. Let $F$ be a CNF formula containing clauses $C_{1}=x_{1} \vee x_{2}, C_{2}=$ $\bar{x}_{2} \vee x_{3}, C_{3}=\bar{x}_{2} \vee x_{4}, C_{4}=x_{1} \vee \bar{x}_{3} \vee \bar{x}_{4}$ (and some other clauses). Suppose that the decision assignment $x_{1}=0$ has been made in $F$ and the Boolean Constraint Propagation (BCP) procedure is invoked after that. Then the assignment $x_{2}=1$ has to be made to satisfy $C_{1}$. This leads to derivation of assignments $x_{3}=1$ and $x_{4}=1$ required to satisfy $C_{2}$ and $C_{3}$ respectively. At this point, a conflict occurs because clause $C_{4}$ becomes unsatisfiable. Denote by $F^{\prime}$ the set of clauses $\left\{C_{1}, C_{2}, C_{3}, C_{4}\right\}$ i.e. $F^{\prime}$ is the subformula of $F$ responsible for the conflict.

The conflict clause is derived from $F^{\prime}$ as follows [13]. The set $\operatorname{Vars}\left(F^{\prime}\right)$ can be partitioned into subsets $X_{1}=\left\{x_{2}, x_{3}, x_{4}\right\}$ and $X_{2}=\left\{x_{1}\right\}$. The set $X_{1}$ consists of the variables on which clauses of $F^{\prime}$ are resolved when producing the conflict clause. Variables of $X_{1}$ are used in the order opposite to the one in which these variables were assigned. First, clause $C_{4}$ is resolved with $C_{3}$ on variable $x_{4}$ producing resolvent $R_{1}=\bar{x}_{2} \vee x_{1} \vee \bar{x}_{3} . R_{1}$ is resolved with $C_{2}$ on variable 
$x_{3}$ producing the resolvent $R_{2}=\bar{x}_{2} \vee x_{1}$. Clauses $R_{2}$ and $C_{1}$ are resolved on variable $x_{2}$ producing the conflict clause $x_{1}$ (consisting only of variables of $X_{2}$ ).

Now we show that the same conflict clause is produced by the BPE procedure when existentially quantifying away the variables of $X_{1}$ from $F^{\prime}$. The necessary boundary points can be trivially produced from the partial assignment $\boldsymbol{q}$ of variables of $F$ leading to the conflict. That is $\boldsymbol{q}$ is the assignments to the variables of $\operatorname{Vars}\left(F^{\prime}\right)$ and so $\boldsymbol{q}$ is equal to $\left(x_{1}=0, x_{2}=1, x_{3}=1, x_{4}=1\right)$. By construction, $\boldsymbol{q}$ falsifies the unsatisfiable clause $C_{4}=x_{1} \vee \bar{x}_{3} \vee \bar{x}_{4}$. This means, in particular, that $\boldsymbol{q}$ is an $\bar{x}_{4}$-boundary point of $F^{\prime}$. The point $\boldsymbol{q}^{*}=\operatorname{flip}\left(\boldsymbol{q}, x_{4}\right)$ falsifies only clause $C_{3}=\bar{x}_{2} \vee x_{4}$ of $F^{\prime}$. So $\boldsymbol{q}^{*}$ is an $x_{4}$-boundary point of $F^{\prime}$. Adding the resolvent $R_{1}=\bar{x}_{2} \vee x_{1} \vee \bar{x}_{3}$ of $C_{4}$ and $C_{3}$ to $F^{\prime}$ eliminates $\boldsymbol{q}$ and $\boldsymbol{q}^{*}$ as $l\left(x_{4}\right)$ boundary points. Adding $R_{1}$ also quantifies away the variable $x_{4}$ of $F^{\prime}$ (no more clauses of $F^{\prime}$ can be resolved on $x_{4}$ ). Then $C_{3}$ and $C_{4}$ are eliminated from $F^{\prime}$ (as containing variable $x_{4}$ ) and the assignment $x_{4}=1$ is removed from $\boldsymbol{q}$.

Now $\boldsymbol{q}=\left(x_{1}=0, x_{2}=1, x_{3}=1\right)$ falsifies only the resolvent $R_{1}$. So $\boldsymbol{q}$ is a $\bar{x}_{3^{-}}$ boundary point for $F^{\prime}$. The point $\boldsymbol{q}^{*}=f \operatorname{fip}\left(\boldsymbol{q}, x_{3}\right)$ falsifies only clause $C_{2}=\bar{x}_{2} \vee x_{3}$ of $F^{\prime}$. So $\boldsymbol{q}^{*}$ is an $x_{3}$-boundary point of $F^{\prime}$. Adding the resolvent $R_{2}=\bar{x}_{2} \vee x_{1}$ of $R_{1}$ and $C_{2}$ to $F^{\prime}$ eliminates $\boldsymbol{q}$ and $\boldsymbol{q}^{*}$ as $l\left(x_{3}\right)$-boundary points. Adding $R_{2}$ to $F^{\prime}$ also quantifies away the variable $x_{3}$ from $F^{\prime}$. The clauses $R_{1}$ and $C_{2}$ are removed from $F^{\prime}$ and the assignment $x_{3}=1$ is removed from $\boldsymbol{q}$.

Points $\boldsymbol{q}=\left(x_{1}=0, x_{2}=1\right)$ and $\boldsymbol{q}^{*}=\operatorname{flip}\left(\boldsymbol{q}, x_{2}\right)$ falsify only the resolvent $R_{2}$ and clause $C_{1}=x_{1} \vee x_{2}$ respectively. Adding the resolvent $x_{1}$ of $R_{2}$ and $C_{1}$ on $x_{2}$ to $F^{\prime}$ eliminates $\boldsymbol{q}$ and $\boldsymbol{q}^{*}$ as $l\left(x_{2}\right)$-boundary points. Adding $x_{1}$ also quantifies away the variable $x_{2}$ from $F^{\prime}$. Then $R_{2}$ and $C_{1}$ are removed from $F^{\prime}$ making the resolvent $x_{1}$ the only clause left.

Formal description. Now we give a formal description. (Note that numbering of clauses, variables and resolvents here is different from the example above.) Let $F$ be a CNF formula and $F^{\prime}=\left\{C_{1}, \ldots, C_{k+1}\right\}$ be the set of clauses responsible for a conflict found by the BCP procedure.. We assume that the set $F^{\prime}$ is irredundant i.e. every clause $C_{i}, 1 \leq i \leq k+1$ contributed to the conflict. To simplify the notation we make the following two assumptions. First, BCP derived assignments from $C_{1}, \ldots, C_{k}$ in the numbering order (i.e. first from $C_{1}$, then from $C_{2}$ and so on). $C_{k+1}$ is the falsified clause (the cause of the conflict). Second, we assume that clause $C_{i}, i=1, \ldots, k$ contains the positive literal of $x_{i}$ and the assignment $x_{i}=1$ was derived from $C_{i}$ during BCP.

A conflict clause is obtained by resolving clauses of $F^{\prime}$ in the reverse order. That is, first $C_{k+1}$ is resolved with $C_{k}$ on variable $x_{k}$. Denote their resolvent as $R_{k}$. Then $R_{k}$ is resolved with $C_{k-1}$ on variable $x_{k-1}$ producing resolvent $R_{k-1}$. Eventually, resolvent $R_{2}$ is resolved with $C_{1}$ on variable $x_{1}$ producing resolvent $R_{1}$ which is a conflict clause. (Note that $R_{i}, 1 \leq i \leq k$, has to contain literal $\bar{x}_{i}$. Otherwise, no clause $C_{j}, j>i$ contains literal $\bar{x}_{i}$, which implies that the assignment $x_{i}=1$ derived from $C_{i}$ has not contributed to the conflict. This contradicts our assumption that $F^{\prime}$ consists only of clauses responsible for the conflict. Clause $C_{k+1}$ has to contain $\bar{x}_{k}$ for the same reason.) The set $X=\operatorname{vars}\left(F^{\prime}\right)$ can be partitioned into the set $X_{1}=\left\{x_{1}, \ldots, x_{k}\right\}$ and 
$X_{2}=X \backslash X_{1}$. That is, $X_{1}$ consists of the variables on which clauses of $F^{\prime}$ were resolved. The conflict clause $R_{1}$ consists of the variables of $X_{2}$.

Let us show that the same conflict clause $R_{1}$ is produced by the BPE procedure by quantifying away the variables of $X_{1}$ from $F^{\prime}$. Let $\boldsymbol{q}$ be the assignments to the variables of $X$ made before the conflict occurred. The main idea is to show that the BPE procedure uses the same resolutions (as the conflict clause generation procedure above) to eliminate boundary points obtained from $\boldsymbol{q}$.

By construction, $\boldsymbol{q}$ falsifies only clause $C_{k+1}$ of $F^{\prime}$. (Clause $C_{i}, 1 \leq i \leq k$ is satisfied by the assignment $x_{i}=1$ derived from $C_{i}$ during BCP). Then $\boldsymbol{q}$ is an $\bar{x}_{k}$-boundary point of $F^{\prime}$. (Clause $C_{k+1}$ contains $\left.\bar{x}_{k}\right)$. The point $\boldsymbol{q}^{*}=\operatorname{flip}\left(\boldsymbol{q}, x_{k}\right)$ falsifies only the clause $C_{k}$ (by construction it is the only clause that has literal $x_{k}$ ). So $\boldsymbol{q}^{*}$ is an $x_{k}$-boundary point of $F^{\prime}$. Adding to $F^{\prime}$ the resolvent $R_{k}$ of $C_{k+1}$ and $C_{k}$ on $x_{k}$ eliminates $\bar{x}_{k}$-boundary point $\boldsymbol{q}$ and $x_{k}$-boundary point $\boldsymbol{q}^{*}$. This concludes processing variable $x_{k}$ of $F^{\prime}$ (only clauses $C_{k+1}$ and $C_{k}$ can be resolved on $x_{k}$ ).

After removing $C_{k+1}$ and $C_{k}$ from $F^{\prime}$ (as the clauses depending on variable $x_{k}$ ) and removing the assignment to $x_{k}$ from $\boldsymbol{q}$, one reproduces the same situation as above with respect to variable $x_{k-1}$. Now $\boldsymbol{q}$ falsifies only the clause $R_{k}$ of $F^{\prime}$. Since $R_{k}$ contains $\bar{x}_{k-1}, \boldsymbol{q}$ is a $\bar{x}_{k-1}$-boundary point of $F^{\prime}$. The point $\boldsymbol{q}^{*}=\operatorname{flip}\left(\boldsymbol{q}, x_{k-1}\right)$ falsifies only the clause $C_{k-1}$ of $F^{\prime}$ and so it is an $x_{k-1}$-boundary point of $F^{\prime}$. Adding to $F^{\prime}$ the resolvent $R_{k-1}$ of $C_{k-1}$ and $R_{k}$ on $x_{k-1}$ eliminates $\boldsymbol{q}$ as a $\bar{x}_{k-1}$-boundary point and $\boldsymbol{q}^{*}$ as an $x_{k-1}$-boundary point. This concludes processing variable $x_{k-1}$. After the BPE procedure is done with all the variables of $X_{1}, F^{\prime}$ reduces to resolvent $R_{1}$ that is exactly the clause generated by the conflict clause generation procedure described above.

\section{BPE-SAT and DP procedure}

In this subsection, we use formulas $F(r, m)$ described in Section 9 to compare the implementation of BPE-SAT meant for narrow formulas (see Section 7) and the DP procedure [6].

The reason is twofold. First, the DP procedure and the BPE procedure of BPE-SAT are very similar. The only difference is that when quantifying away variable $x_{i}$, the DP procedure adds all the resolvents on variable $x_{i}$ while the BPE procedure adds only resolvents (on variable $x_{i}$ ) that eliminate symmetric $x_{i}$-boundary and $\bar{x}_{i}$-boundary points. So it is interesting to see how beneficial this reduction in the number of generated resolvents is. Second, the DP procedure is exponential only in formula width [7]. So one may think that the good performance of BPE-SAT on formulas $F(r, m)$ for small values of $r$ trivially follows from the theory of [7].

\footnotetext{
1 To compute the approximate ratio for the formula $F(4,80)$ (with ordering width 92 ) we used the percentage of variables resolved out by the time the DP procedure was aborted (0.09\%).
} 
Table 6. DP procedure and BPE-SAT

\begin{tabular}{|l|l|l|l|l|l|}
\hline$(r, m)$ & $\begin{array}{l}(\text { vars, } \\
\text { cls. } \\
\times 1000\end{array}$ & $\begin{array}{l}\text { wi- } \\
\text { dth }\end{array}$ & $\begin{array}{l}\text { DP } \\
(\text { s. })\end{array}$ & $\begin{array}{l}\text { BPE- } \\
\text { SAT } \\
(\text { s. })\end{array}$ & $\begin{array}{l}\text { ratio } \\
\text { DP } / \\
\text { BPE-SAT }^{1}\end{array}$ \\
\hline \multirow{2}{*}{$(2,1000)$} & $(13,34)$ & 8 & 10.3 & 4.5 & 2.3 \\
\cline { 3 - 6 } & 11 & 122 & 4.0 & 30.5 \\
\hline$(3,200)$ & \multirow{2}{*}{$(7,20)$} & 16 & 22.1 & 6.4 & 3.5 \\
\cline { 3 - 6 } & & 30 & 14,150 & 6.0 & 2,358 \\
\hline$(4,80)$ & $(12,34)$ & 29 & 14,408 & 21.6 & 667 \\
\cline { 3 - 6 } & & 92 & $>3 \times 10^{5}$ & 90.2 & $\approx 3.7 \times 10^{6}$ \\
\hline
\end{tabular}

Some experimental results are given in Table 6. (Our implementation of the DP procedure was reasonably efficient. In particular, no resolvent was generated if it was subsumed by an existing clause of the formula.) For each of the three formulas of Table 6 we used two variable orderings (from inputs to outputs and vice versa) that had different width. The first two columns show the value of parameters $(r, m)$ and the number of variables and clauses for each of the three formulas. The values of width are shown in the third column. The next two columns give the runtime of the DP procedure and BPE-SAT (in seconds). The last column shows the ratio of runtimes.

One can make the following two conclusions based on the results of Table 6 . First, the DP procedure is not competitive with CDCL SAT-solvers on formulas $F(r, m)$ even for small values of $r$. For example, it took about 4 hours for the DP procedure to complete $F(4,80)$ (for the most favorable variable ordering) while Minisat and Picosat solved it in 9.8 and 13.2 seconds respectively. Second, although both DP procedure and BPE-SAT are sensitive to the formula width, the sensitivity of the DP procedure is dramatically higher. In particular, in 300,000 seconds it was able to process only 11 variables out of 12 thousands for the formula $F(4,80)$ for the variable ordering with the width of 92 . 